\title{
ARTICLE
}

\section{Monte Carlo calculations with MCNPX and GEANT4 for general shielding study - Application to a proton therapy center}

\author{
Thibault Vanaudenhove ${ }^{\mathrm{a}}$, Frédéric Stichelbaut ${ }^{\mathrm{b}}$, Alain Dubus ${ }^{\mathrm{a}}$, Nicolas Pauly $^{\mathrm{a}}$ and Valérie De Smet ${ }^{\mathrm{c}}$ \\ ${ }^{a}$ Université Libre de Bruxelles, 50 avenue F.D. Roosevelt, 1050 Brussels, Belgium; ${ }^{b}$ Ion Beam Applications SA, 3 Chemin du \\ Cyclotron, 1348 Louvain-la-Neuve, Belgium; ' Institut de Recherche de l'Institut Supérieur Industriel de Bruxelles, 150 rue Royale, \\ 1000 Brussels, Belgium.
}

\begin{abstract}
Monte Carlo codes have become an essential tool for studying the radiation protection of particle accelerator facilities such as a proton therapy center. The MCNPX code is well adapted for shielding study but the GEANT4 toolkit is also a suitable solution. Benchmark simulations - secondary-particle production and attenuation - and shielding calculations for a proton therapy center show that both codes provide results in good agreement.
\end{abstract}

Keywords: MCNPX; GEANT4; shielding study; neutron source; attenuation law; proton therapy

\section{Introduction}

Over the last few decades, high-energy proton beams have been increasingly used for medical applications [1]. However, exposure to radiation may injure those standing near the target or around the beam production installation. Indeed, the interaction of a $230-\mathrm{MeV}$ proton beam with matter produces high-energy neutrons up to $230 \mathrm{MeV}$ and photons up to $10 \mathrm{MeV}$ [2]. The risk incurred through exposure can be quantified by using sophisticated numerical tools such as Monte Carlo (MC) radiation transport codes.

The MCNPX [3] and FLUKA [4] codes are often used for shielding study $[5,6]$ but the GEANT4 code $[7,8]$ is also a suitable solution [9]. This toolkit allows free definition of complex geometries, scoring options and physical parameters. However, the choice of physical parameters must be carefully checked to avoid inconsistent results.

It is precisely the aim of this paper to compare MCNPX (version 2.5.0) and GEANT4 (version 9.6) calculations of the secondary particle production and attenuation through a shield (Sections 2 and 3), but also more complex MCNPX and GEANT4 simulations of the dose attenuation through the concrete walls of a proton therapy center (Section 4). Indeed, this work aims to be a preliminary study for a comparison with experimental evaluation of the dose in a proton therapy facility.

\footnotetext{
*Corresponding author. Email: thibault.vanaudenhove@ulb.ac.be
}

\section{Secondary particle production}

\subsection{Source and target}

In a proton therapy center, protons from the initial beam can directly interact with components (cyclotron, dipoles and quadrupoles) composed of various materials such as Nickel, Copper and Tantalum. In this part of the work, a $230-\mathrm{MeV}$ proton beam impinging on a thick cylindrical target (cylinder diameter $\approx$ cylinder length $>$ proton range) composed of these particular materials is considered.

\subsection{MCNPX and GEANT4 physics setting}

The high-energy neutron and photon emission from the proton interaction with matter is mainly due to nuclear reactions. In MC codes, various physical models causing the secondary emission, i.e. for the intranuclear cascade (INC) and evaporation stages, have been implemented. In this work, the MCNPX simulations were performed using the Bertini cascade model and the Dresner evaporation model [3]. For the GEANT4 simulations, the Bertini-like cascade model and the Binary Intranuclear Cascade (BIC) model, both including a pre-equilibrium stage and a WeisskopfEwing evaporation model, were considered [10].

\subsection{Simulations of neutron and photon multiplicities}

The neutron and photon multiplicities (number of emitted particles divided by the number of incident particles) are represented in Figure $\mathbf{1}$ and Figure 2, respectively. Figure 1 shows that the neutron production increases with the target atomic number and the proton energy. The GEANT4 calculations using the Bertini-like 
and the BIC models show a similar neutron production compared to MCNPX simulation results, with a maximum discrepancy of $15 \%$ for Nickel and Tantalum. For Copper, the multiplicity calculated using the Bertini-like and the BIC models is underestimated by $30 \%$ and $40 \%$, respectively, compared to MCNPX simulation results.

In most cases, using the GEANT4 Bertini-like model gives rise to a neutron production $20 \%$ larger than that using the BIC model. The Bertini-like model is consequently more appropriate for a conservative shielding study using the GEANT4 toolkit.

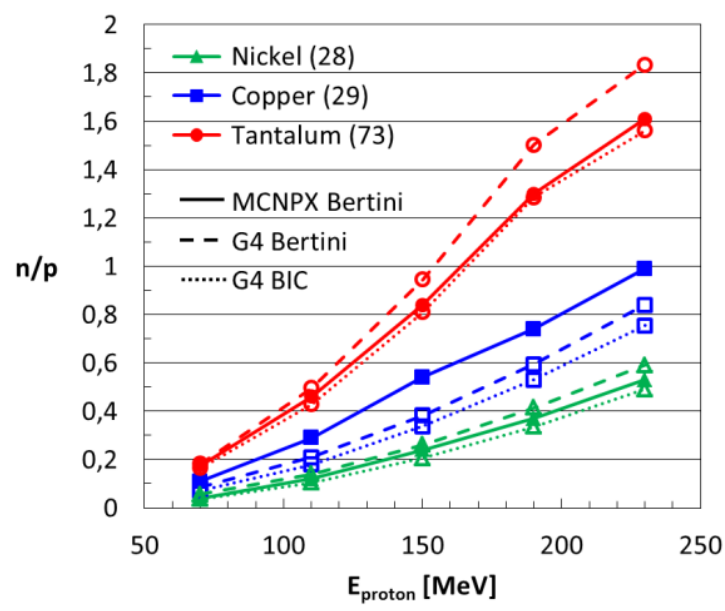

Figure 1. Neutron multiplicity calculated using MCNPX (Bertini model) and GEANT4 (Bertini-like and BIC models).

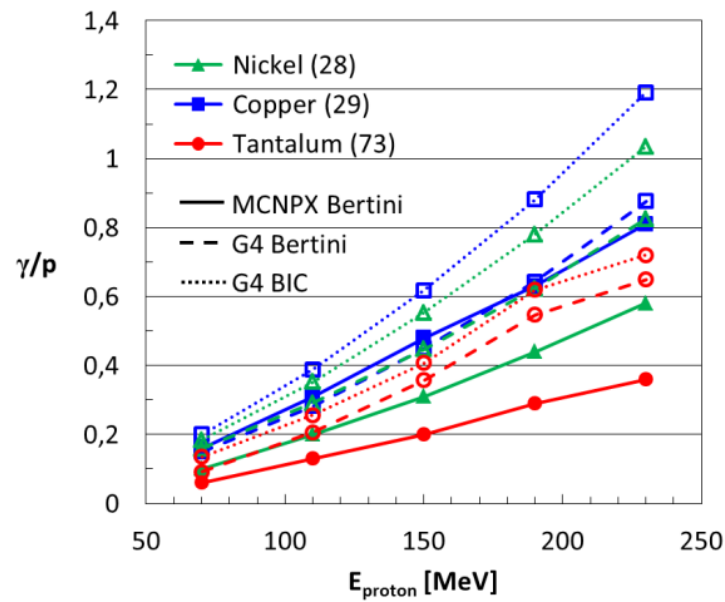

Figure 2. Photon multiplicity calculated using MCNPX (Bertini model) and GEANT4 (Bertini-like and BIC models).

In Figure 2, GEANT4 calculation results using the Bertini-like model and the BIC model show a photon production larger than that obtained from MCNPX calculations, up to a factor of 2 for Tantalum. As photon emission occurs at each interaction stage (cascade, evaporation and other intermediate stages), it is difficult to relate this overproduction to a specific model. This difference must therefore be considered as a phenomenological effect. However, it is well known that the photon dose is always less than $10 \%$ of the neutron dose for shield with sufficient thickness [11]. In the context of this work, this implies that discrepancies on the photon production are not of interest and only the neutron component is considered below.

\subsection{Comparison between neutron yield estimations from GEANT4 simulations and from experiments}

In a proton therapy center, the degrader is usually composed of graphite (see Section 4.1). Figure 3 shows calculations using GEANT4 and the experimental results from Meier et al. [12] of the double differential neutron yield for a $256-\mathrm{MeV}$ proton beam impinging on a Carbon target. In forward directions, the calculation results using the BIC and the Bertini-like models are in good agreement with experiments, with a slight overall overestimation for both. In backward directions, the neutron yield calculated using the BIC model is very similar to experiments while using the Bertini-like model gives rise to a larger high-energy component. Nevertheless, as before, the Bertini model is usually considered because of providing conservative results.

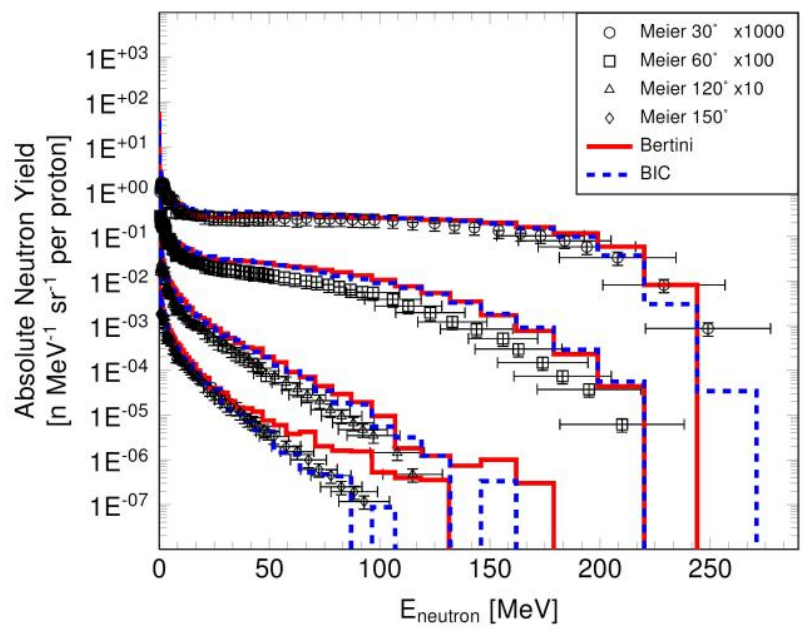

Figure 3. Double differential neutron yield for a $256-\mathrm{MeV}$ proton beam impinging on a Carbon target. Comparison of GEANT4 calculations using the Bertini-like and the BIC models with experimental results from Meier et al. [12].

\section{Neutron attenuation}

\subsection{Sphere model}

For the study of the neutron dose attenuation through a shield, a concrete sphere (density of $2.3 \mathrm{~g} / \mathrm{cm}^{3}$ ) with an inner radius of $1 \mathrm{~m}$ is considered; the source is a $230-\mathrm{MeV}$ proton beam and the target is a thick cylinder.

The effective dose is the protection quantity to be calculated with simulation codes to estimate radiological safety of facilities. Since this quantity cannot be directly evaluated experimentally and this work presents preliminary results for a comparison study between dose calculations and measurements in a proton therapy center, the ambient dose equivalent $\mathrm{H}^{*}(10)$ is used instead. The ambient dose equivalent is computed (conversion coefficients from ICRP 74 [13]) every $20 \mathrm{~cm}$ in the shield and for 18 angles of 10-degree aperture. Variance-reduction techniques based on 
geometry splitting and Russian roulette are used to keep the statistical uncertainties below 3\% (1 sigma).

\subsection{MCNPX and GEANT4 physics setting}

The neutron transport also depends on physical models implemented in MC codes. In MCNPX and GEANT4, tabulated cross-sections can be used for neutron energies below $20 \mathrm{MeV}$ (up to $150 \mathrm{MeV}$ in MCNPX when available) while different specific models are used for larger energies. Recently, a new physics list called "Shielding" has been implemented in GEANT4, which considers tabulated cross-sections and the Bertini-like model. This physics list is considered in all subsequent simulations.

\subsection{Simulation results}

In the considered spherical geometry, $\mathrm{H}^{*}(10)$ is assumed to be proportional to $1 / \mathbf{r}^{2}$, where $\mathbf{r}$ is the distance from the target. Figure 4 shows GEANT4 calculations of the ambient dose equivalent (multiplied by $\mathbf{r}^{2}$ ) due to neutrons produced by a $230-\mathrm{MeV}$ proton beam impinging on a Nickel target. In forward directions, $H^{*}(10) . \mathbf{r}^{2}$ decreases exponentially while in backward directions, two attenuation lengths, computed as "Tenth-Value Lengths" (TVL), can be defined [8]: in the first meter of the shield due to low-energy neutrons (TVLO), and at deeper depth due to more penetrating high-energy neutrons, where an equilibrium is reached (TVLe).

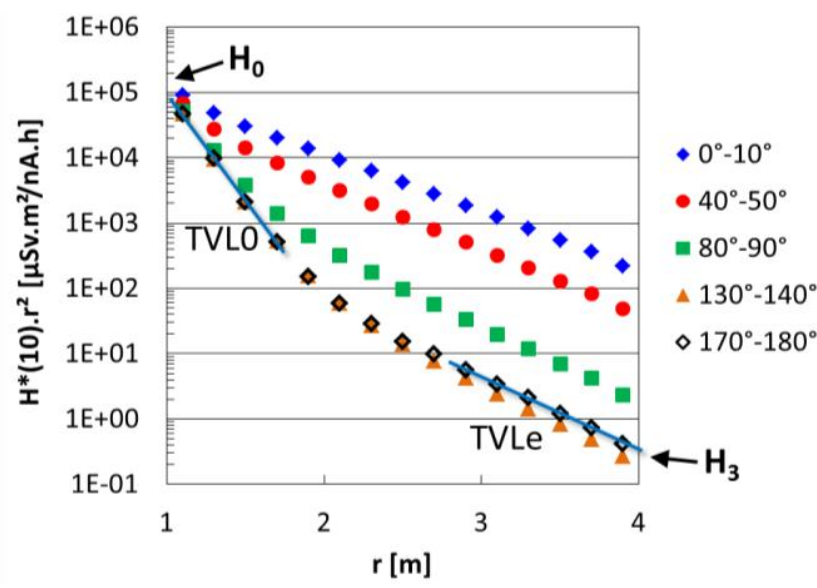

Figure 4. Ambient dose equivalent in concrete as a function of the distance from the target (r) for a $230-\mathrm{MeV}$ proton beam impinging on a Nickel target, calculated using GEANT4.

\subsubsection{Attenuation lengths}

In Figure 5, the values of TVLO and the TVLe calculated using MCNPX and GEANT4 are compared for a Nickel target. As expected in forward directions, both attenuation lengths are similar, while for angles larger than 45 degrees, the TVLe's are larger than the TVL0's. Both codes give similar results except for TVLO at forward angles and for TVLe at backward angles where discrepancies reach $10 \%$ and $20 \%$, respectively.

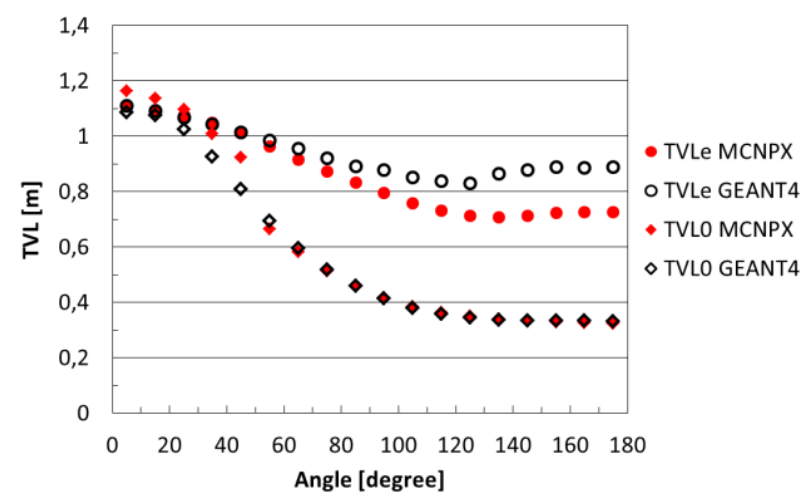

Figure 5. Tenth-Value Lengths in concrete in the first meter of the shield (TVL0) and at deep depth (TVLe) for a $230-\mathrm{MeV}$ proton beam impinging on a Nickel target.

\subsubsection{Dose before the concrete shield}

Calculations of the ambient dose equivalent just before the concrete shield $\left(\mathrm{H}_{0}\right.$ in Figure 4$)$ are shown in Figure 6 for various target materials: tissue (soft ICRP), Nickel and Tantalum. Both codes give very similar results for tissue, with a maximum discrepancy of $25 \%$. For Nickel and Tantalum, $\mathrm{H}_{0}$ values provided by GEANT4 simulations are larger than values from MCNPX calculations up to $35 \%$ for Nickel and $50 \%$ for Tantalum. This is consistent with results shown in Figure 1: the neutron multiplicity calculated using the GEANT4 Bertini-like model is larger than that provided by MCNPX for these materials.

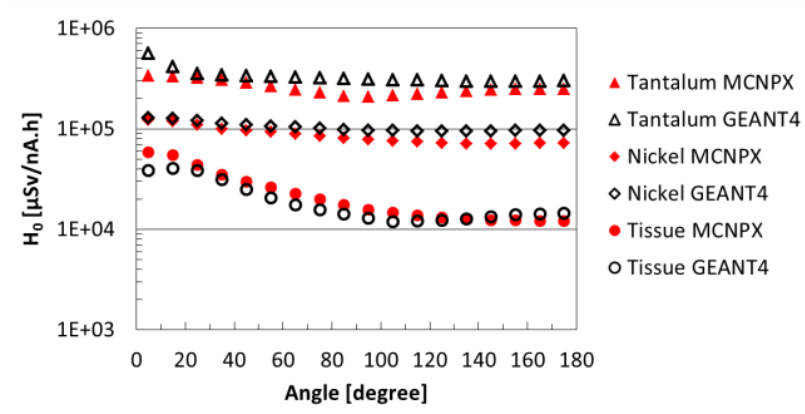

Figure 6. Ambient dose equivalent just before the concrete shield $\left(\mathrm{H}_{0}\right)$ for different target materials. The incident proton energy is $230 \mathrm{MeV}$.

\subsubsection{Dose at $3 \mathrm{~m}$ depth in concrete}

Figure 7 shows $\mathrm{H}^{*}(10)$ calculated using GEANT4 and MCNPX at $3 \mathrm{~m}$ depth in concrete $\left(\mathrm{H}_{3}\right.$ in Figure 4$)$ for the same target materials as considered above. In forward directions, the dose is independent of the target material, while in backward directions, the dose increases with the target atomic number. At forward angles, doses calculated using GEANT4 are two times smaller than the values provided by MCNPX, while at backward angle, values from the GEANT4 simulations are larger than that provided by MCNPX, up to a factor of 3 for tissue. This difference may be due to the worse performance of the GEANT4 Bertini-like model for backward directions (see Figure 3). 


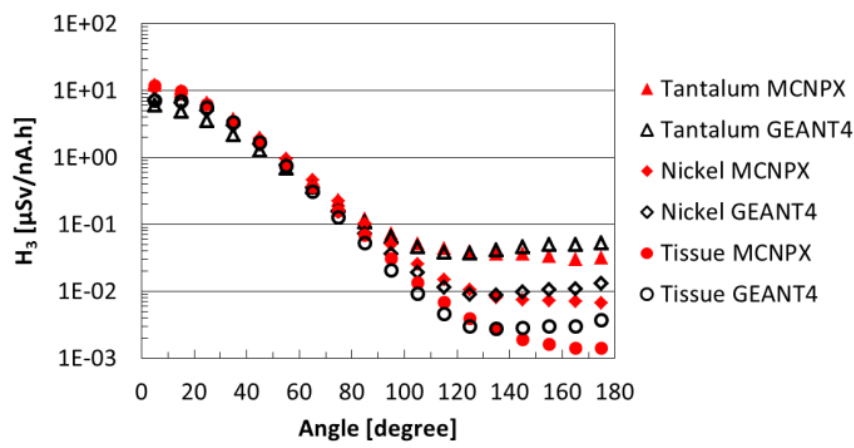

Figure 7. Ambient dose equivalent at $3 \mathrm{~m}$ depth in concrete $\left(\mathrm{H}_{3}\right)$ for different target materials. The incident proton energy is $230 \mathrm{MeV}$.

\section{Shielding study of a proton therapy center}

\subsection{Center description and source definition}

In this work, a typical proton therapy center from Ion Beam Applications (IBA, SA) is considered. The cyclotron room includes a $230-\mathrm{MeV}$ cyclotron, a graphite degrader (variable thickness for selecting beam energy between $70 \mathrm{MeV}$ and $230 \mathrm{MeV}$ ), an Energy Selection System (ESS, including dipole and quadrupole magnets), concrete walls, floors and roofs. Figure 8 shows the cyclotron room, the fixed-beam room and one gantry room, simulated using GEANT4.

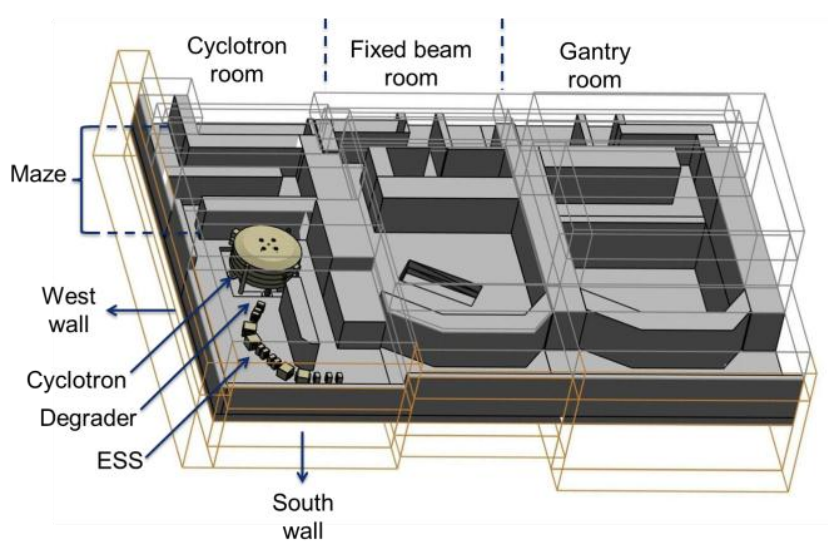

Figure 8. IBA protontherapy center simulated using GEANT4. Cyclotron, ESS, walls and floors are solid, roofs and outer walls are transparent.

In the cyclotron room, the main neutron sources are due to the interaction of the beam with the degrader (a thick cylindrical target is considered) and the loss of the beam inside the cyclotron. The quantification of the latter is quite difficult and it is thus modeled by a proton beam impinging on three Copper targets localized at the two counter-D's and the septum positions.

Based on data from IBA, a $40 \%$ extraction efficiency and a $60 \%$ beam loss inside the cyclotron $(10 \%$ from each counter-D and $40 \%$ from the septum) are assumed. An extracted annual beam workload of 82200 nA.h is considered.

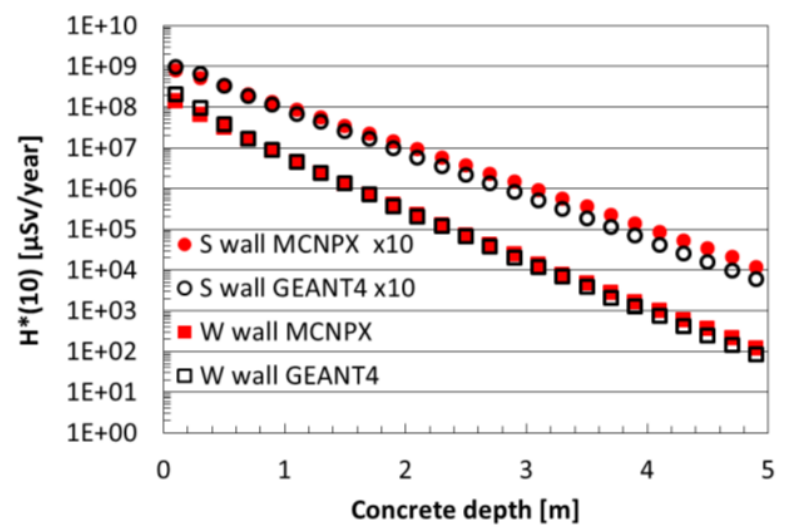

Figure 9. Ambient dose equivalent in the south and west walls of the cyclotron room. For clarity, the dose in the south wall is multiplied by a factor of 10 .

\subsection{Simulation results}

\subsubsection{Dose in west and south walls}

Figure 9 shows doses calculated using MCNPX and GEANT4 in the west and south walls of the cyclotron room, without considering the ESS. The neutrons from the degrader are the main contributors to this dose. Because these walls are in front of the beam direction, the dose follows a one-attenuation-length exponential. This behavior was already shown in Figure 4. Both codes give similar results, although at a $5 \mathrm{~m}$ depth, the discrepancies can reach $40 \%$ and $95 \%$ for the west and south walls, respectively.

\subsubsection{Dose in the maze walls}

Figure 10 shows doses in the three maze walls of the cyclotron room, calculated using MCNPX and GEANT4. Discrepancies between the two codes are globally of a factor of 2, but reach a factor of 3 for some points. However, these discrepancies are quite reasonable with regards to the physics implemented in the codes and the geometry complexity.

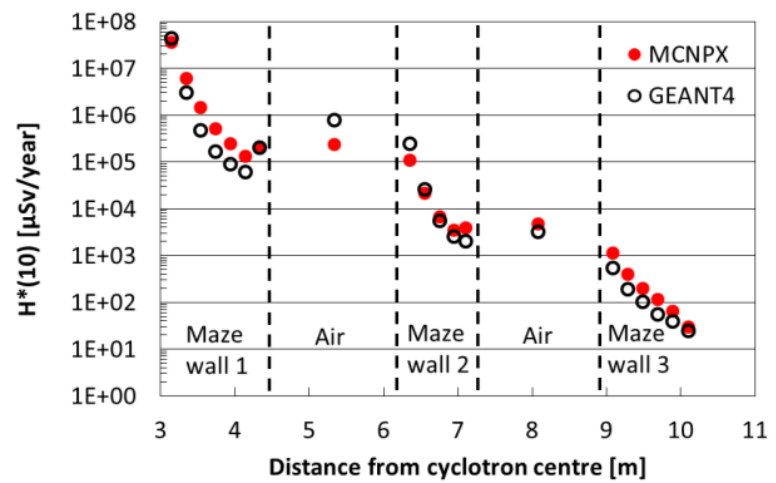

Figure 10. Ambient dose equivalent in the maze walls of the cyclotron room.

\section{Conclusion}

The GEANT4 toolkit satisfies reasonably the three main requirements of a numerical tool to be suitable for a shielding study of a proton therapy center: accurate or conservative secondary-yield production (compared with experimental data), accurate transportation through a 
shielding wall (compared with MCNPX calculations), and the combination of the two previous conditions for a more complex geometry (compared with MCNPX calculations). A detailed comparison study between MC calculations and measurements of the ambient dose equivalent behind the shielding walls and in the mazes will be done in a future work.

\section{Acknowledgements}

T. Vanaudenhove wishes to thank Association Vinçotte Nuclear (AVN) for their research funding.

\section{References}

[1] A.R. Smith, Proton therapy, Phys. Med. Biol. 51 (2006), pp. R491-R504.

[2] A. Ferrari, M. Ferrarini and M. Pelliccioni, Secondary particle yields from $400 \mathrm{MeV} / \mathrm{u}$ carbon ion and $250 \mathrm{MeV}$ proton beams incident on thick targets, Nucl. Instrum. Meth. Phys. Res. B 269 (2011), pp. 1474-1481.

[3] Los Alamos National Laboratory, MCNPX 2.6 manual, LA-CP-07-1473 (2008).

[4] A. Fassò, A. Ferrari, J. Ranft and P.R. Sala, FLUKA: A Multi-Particle Transport Code, CERN-2005-10, INFN/TC_05/11, SLAC-R-773, (2005).

[5] J.-W. Kim, J.-W. Kwon and J. Lee, Design of radiation shielding for the proton therapy facility at the National Cancer Center in Korea, Radiat. Prot. Dosim. 115 (2005), pp. 271-275.
[6] S. Agosteo, Radiation protection constraints for use of proton and ion accelerators in medicine, Radiat. Prot. Dosim. 137 (2009), pp. 167-186.

[7] S. Avery, C. Ainsley, R. Maughan and J. McDonough, Analytical shielding calculations for proton therapy facility, Radiat. Prot. Dosim. 131 (2008), pp. 167-179.

[8] S. Agostinelli, J. Allison, K. Amako et al., GEANT4 - a simulation toolkit, Nucl. Intrum. Meth. A 506 (2003), pp. 250-303.

[9] J. Allison, K. Amako, J. Apostolakis et al., GEANT4 developments and applications, IEEE Trans. Nucl. Sci. 53 (2006), pp. 270-278.

[10]J. Apostolakis, A.V. Ivantchenko, V.N. Ivanchenko, M. Kossov, J.-M. Quesada and D. H. Wright, GEANT4 simulation of nuclear spallation reactions, Proc. International Topical Meeting on Nuclear Research Applications and Utilization of Accelerators, Vienna, 4-8 May, 2009, (2010), SM/SR-10.

[11]S. Agosteo, M. Magistris and M. Silari, Shielding of proton accelerators, Radiat. Prot. Dosim. 146 (2011), pp. 414-424.

[12]M.M. Meier, C.A. Goulding, G.L. Morgan and J.L. Ullmann, Neutron yields from stopping- and near-stopping-length targets for $256-\mathrm{MeV}$ protons, Nucl. Sci. Eng. 104 (1990), pp. 339-363.

[13] International Commission on Radiological Protection, Conversion Coefficients for Use in Radiological Protection against External Radiation, ICRP Publication 74, (1997). 